\title{
Numerical analysis of lateral movement of a metal strip during cold rolling
}

\author{
T. Tarnopolskaya D. J. Gates F. R. de Hoog* \\ W. Y. D. Yuen A. Dixon ${ }^{\dagger}$
}

(Received 8 August 2003; revised 31 March 2004)

\begin{abstract}
A model and numerical algorithm for the analysis of lateral movement of a metal strip during cold rolling are presented. The model includes a simplified description of the physical processes responsible for strip lateral movement, such as plastic deformation of the strip, elastic deformation of rolls, deformation and dynamics of the strip outside the plastic reduction region. The study is motivated by the operational problem experienced in the metal rolling industry. $\mathrm{Nu}-$ merical simulations reveal possible mechanisms of strip track-off and identify issues inviting further study.
\end{abstract}

${ }^{*}$ CSIRO Mathematical \& Information Sciences, Australia. mailto:tanya.tarnopolskaya@csiro.au

${ }^{\dagger}$ BHP Steel Research Laboratories

See http://anziamj.austms.org.au/V45/CTAC2003/Tarn for this article, (C) Austral. Mathematical Soc. 2004. Published 12 April 2004, amended April 20, 2004. ISSN 1446-8735 


\section{Contents}

1 Introduction

C174

2 Mathematical model

C175

2.1 Plastic deformation of the strip in the roll bite . . . . . C176

2.2 Elastic deformation of the roll stack . . . . . . . . . C C177

2.3 Rolling model and its structure . . . . . . . . . . . C177

2.4 Strip dynamics . . . . . . . . . . . . . . . . . . . . . C C179

2.5 Steady-state solution for strip off-centre . . . . . . . . C180

3 Numerical examples and discussion

C182

3.1 Dynamics of an unconstrained strip . . . . . . . . . . C C182

3.2 Steady state solution for a constrained strip . . . . . . . C183

References

C185

\section{Introduction}

Rapid lateral movement of a metal strip during rolling is an operational problem that leads to serious consequences, such as strip chew-ups and mill crashes. The fact that strip tracking failure occurs very rapidly poses major problems for experimental study of the phenomenon and invites mathematical modelling. The problem became more pronounced with increased rolling speed and attracted increasing attention of researchers over the last decade. Several attempts to model the strip track-off phenomenon are reported in the literature $[1,2,3,4]$. However, because of the complexity of the problem, the models either contain assumptions that limit their usefulness [2, 3, 4] or involve empirical parameters that have to be adjusted when rolling conditions vary $[1]$.

In this paper, we develop a model that aims to take into account the 
main physical processes responsible for strip tracking, such as the plastic deformation of the strip in the roll bite, the elastic deformation of the roll stack and the dynamics and deformation of the strip outside the roll bite. This work is an extension of the authors' earlier work [4] that concentrated on the causes of strip track-off within the plastic reduction region and ignored the motion and deformation of the strip outside the roll bite. Because of the complexity of the problem, and with a view to possible future application in an on-line control system, we use simplified models that still adequately describe the physics of the processes involved. The structure of the model and the efficient numerical algorithm are briefly discussed.

The study in this paper concentrates on two cases representing the practical situations where strip track-off could occur:

1. the tail out situation (the rolling of the strip that has free end upstream);

2. the steady-state rolling of the strip that is constrained upstream in a tension producing device.

Numerical simulations reveal that unstable tracking is intrinsic in the tail out rolling under asymmetric conditions. It could also occur for a strip constrained upstream, if the tension applied to the strip is not sufficient to prevent strip buckling. Strip buckling is identified as major cause for strip tracking failure and calls for further investigation.

\section{Mathematical model}

In this section, the constitutive parts of the model of lateral movement of the strip during cold rolling are presented. An overall structure of the proposed model and the efficient numerical procedure are also discussed. 


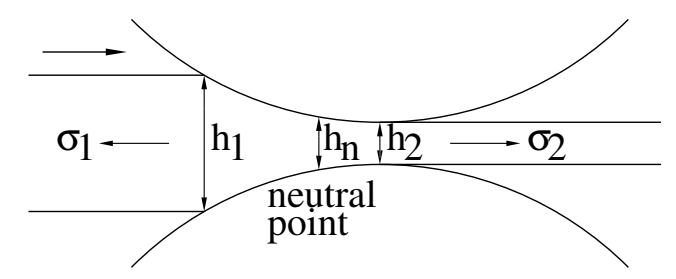

Figure 1: Schematics of a roll bite

\subsection{Plastic deformation of the strip in the roll bite}

In order to describe the vertical force acting on the strip in the roll bite (the so-called roll force), we adopt a conventional approach based on a generalised two-dimensional plastic deformation theory [5, e.g.] that proved to produce sufficiently accurate results in practical rolling situations $[6,7]$. This approach, combined with widely used classical plain strain model by Ford and Bland [8] and well-known Hitchcock formula [9] for a deformed radius of the roll, has the following structure

$$
\begin{array}{r}
f\left(h_{2}, p\right)\left[\left(1-\sigma_{2}\right) \gamma_{1}\left(\phi_{n}, h_{2}, p\right)+\left(1-\sigma_{1}\right) \gamma_{2}\left(\phi_{n}, h_{2}, p\right)\right]=p \\
\left(1-\sigma_{2}\right) \gamma_{3}\left(\phi_{n}, h_{2}, p\right)-\left(1-\sigma_{1}\right) \gamma_{4}\left(\phi_{n}, h_{2}, p\right)=0,
\end{array}
$$

where $p(x)$ is the roll force, $\phi_{n}(x)$ is the neutral angle, $x$ is the co-ordinate across strip width, the functions $f$ and $\gamma_{i}, i=1,4$, depend on the properties of the materials of the rolls and the strip. The system of non-linear algebraic equations (1-2) is usually solved for the roll force $p$ and the neutral angle $\phi_{n}$, while the longitudinal stresses at the entry and the exit $\sigma_{1}(x)$ and $\sigma_{2}(x)$ as well as the exit strip thickness $h_{2}(x)$ (Figure 1) are required as an input. 


\subsection{Elastic deformation of the roll stack}

Half of a roll stack of a four-high mill (we assume symmetry about the strip mid-thickness) is shown in Figure 2a. External forces acting on the system include jack bending forces and roll force on the interface between the work roll and the strip. In modelling the roll stack deformation we have adopted a conventional approach [10] consisting of superposition of deformation due to the bending of the roll axes, calculated using Timoshenko beam theory, and the local surface flattening, which is calculated using Hertzian contact theory. For asymmetric loading and geometry of the mill, the model was derived in the authors' earlier work [4]. By using Green's function solution for roll bending the model was reduced to a system of non-linear algebraic and integral equations.

In [11], we discussed two alternative formulations of this model with regard to the boundary conditions on the interface with rolled strip. It was shown [11] that, given the structure of rolling model coupling, it is more appropriate, despite the common practice, to formulate the roll stack deformation model as a second fundamental boundary value problem and solve it for variation of the roll force $\tilde{p}$ across the strip width using the variation of the exit strip thickness and the mean value of the rolling force as an input, that is

$$
\tilde{p}(x)=\tilde{p}\left(\tilde{h}_{2}(x), \bar{p}\right) .
$$

We make the conventional assumption that the profile of the strip at the entry of the roll bite conforms to the profile of the deformed work roll. Hereafter, a tilde denotes the deviation from the mean value, while a bar denotes a mean value.

\section{$2.3 \quad$ Rolling model and its structure}

A connection of the processes within the reduction mill, described in Sections 2.1 and 2.2, with the processes outside the roll bite is established by 
introducing the boundary condition of strip velocity continuity at the interface between the plastic deformation region and the region outside the roll bite in the form [1]

$$
\tilde{v}_{i}=\omega_{i} x, \quad i=1,2,
$$

where $\omega_{1}$ and $\omega_{2}$ are the angular speeds of the strip at the entry and exit of the roll bite, $\tilde{v}_{1}$ and $\tilde{v}_{2}$ are the deviations of the velocities of the strip from their mean values, $x$ is the co-ordinate across the strip width measured from the centre of the strip. The variations of strip velocities $\tilde{v}_{1}$ and $\tilde{v}_{2}$ are expressed through the variations of the neutral angle and the exit strip thickness using the mass conservation within the roll bite, thus providing the coupling of equations (4) with the equations of plastic deformation of the strip and elastic deformation of the roll stack (Sections 2.1 and 2.2).

In what follows, we call the models described in Sections 2.1 and 2.2, together with the conditions of continuity of strip velocity (4), a "rolling model". This model involves several coupled models and requires iterative solution. A model of a similar structure was analysed in the authors' earlier papers $[11,12]$. It was found that the model can be re-formulated in a form that makes the parts of the model only weakly coupled and therefore an efficient numerical procedure organized. Using the arguments similar to those in [11], the following algorithm is suggested:

1. Calculate $\bar{\phi}_{n}$ and $\bar{p}$ using the roll bite model (1-2) and the known values of $\bar{\sigma}_{1}, \bar{\sigma}_{2}, \bar{h}_{1}, \bar{h}_{2}$;

2. Calculate $\tilde{\phi}_{n}$ and $\tilde{h}_{2}$ from strip velocity continuity conditions (4) together with the condition of mass conservation in the roll bite;

3. Calculate $\tilde{p}$ from the model of the elastic deformation of the roll stack (3);

4. Re-formulate the model of plastic deformation (1-2) as

$$
\sigma_{i}=\sigma_{i}\left(\phi_{n}, h_{2}, p\right), \quad i=1,2,
$$

and solve for $\tilde{\sigma}_{1}, \tilde{\sigma}_{2}, \bar{\phi}_{n}$ and $\bar{p}$. 


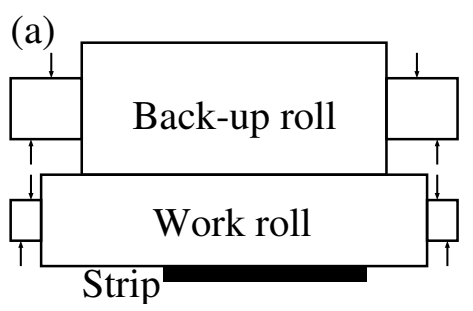

(b)

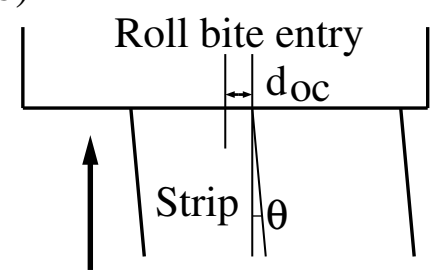

FiguRE 2: Schematics of (a) half of the rolling stack, (b) strip motion.

In principle, Steps 2-4 can be repeated till convergence is achieved. However, in our experience sufficient accuracy is obtained after the first cycle of iterations.

The "rolling model" is used to calculate the moments of tensile stresses acting on the strip at the entry and exit of the roll bite as functions of the asymmetry in loading and mill geometry (including strip off-centre $d_{\mathrm{oc}}$ ) and the rotational speeds of the strip at the entry and exit of the roll bite

$$
M_{i}=M_{i}\left(d_{\mathrm{oc}}, \omega_{1}, \omega_{2}, \text { asymmetry }\right)=\int_{-L_{s}}^{L_{s}} \sigma_{i}(x) h_{i}(x) x d x, \quad i=1,2,
$$

where $L_{s}$ is half strip width. In what follows, for the sake of definiteness we assume that $\omega_{2}=0$.

\subsection{Strip dynamics}

The equations of strip motion can be written in the form [1]

$$
\begin{aligned}
\frac{d \theta}{d t} & =\omega_{1}\left(d_{\mathrm{oc}}, M_{1}\right)-\left.\bar{v}_{1} \kappa\left(d_{\mathrm{oc}}, M_{1}\right)\right|_{\mathrm{entry}}, \\
\frac{d d_{\mathrm{oc}}}{d t} & =v_{R} \theta
\end{aligned}
$$


where $\theta$ is the angle the strip centreline forms with the rolling direction at the entry to the roll bite (Figure $2 b$ ), $\kappa$ is the curvature of the strip centreline, $t$ is the time, $v_{R}$ is the roll speed and $\bar{v}_{1}$ is the mean speed of the strip at the entry of the roll bite. The boundary conditions are

$$
\left.\theta\right|_{t=0}=\theta_{0},\left.\quad d_{\mathrm{oc}}\right|_{t=0}=d_{\mathrm{oc}}^{0} .
$$

For a strip that is free upstream, $\left.\kappa\right|_{\text {entry }}=0$. The rotational speed of the strip $\omega_{1}\left(d_{\text {oc }}, M_{1}\right)$ can be calculated from simultaneous solution of the rolling model (Sections 2.1-2.3) and the condition $M_{1}\left(d_{\mathrm{oc}}, \omega_{1}\right.$, rolling conditions $)=0$.

\subsection{Steady-state solution for strip off-centre}

Consider a strip that is geometrically constrained upstream, at the distance $L$ from the reduction roll so that

$$
\left.u\right|_{z=0}=\left.\frac{\partial u}{\partial z}\right|_{z=0}=0,
$$

where $z$ is the axis along the rolling direction through the centre of the roll with the origin at a distance $L$ from the reduction roll, $u$ is the deflection of the strip in the $x$ direction (positive to the right). These conditions are the simplified representation of the constraints imposed on the strip at the upstream tension device.

At steady-state, the equations of strip motion (7-8) can be written

$$
\begin{aligned}
\omega_{1} & =\left.\kappa\right|_{z=L} \bar{v}_{1}, \\
\theta & =\left.\frac{\partial u}{\partial z}\right|_{z=L}=0 .
\end{aligned}
$$

Using the Bernoulli-Eulerian theory for a bending of strip centreline, the deflections of strip centreline are described by

$$
\frac{\partial^{2} u}{\partial z^{2}}=\frac{M_{1}}{E I}-\frac{G}{E I}(L-z)-\frac{T}{E I}\left(d_{\mathrm{oc}}-u\right),
$$


where $d_{\mathrm{oc}}=\left.u\right|_{z=L}, T=\int_{-L_{s}}^{L_{s}} \sigma_{1} h_{1} d s, I$ is the moment of inertia of the cross-section, $E$ is Young modulus. An extra boundary condition is required to calculate the unknown lateral force $G$. The solution for strip off-centre obtained by solving equation (13) with boundary conditions (10) and (12) is

$$
d_{\mathrm{oc}}=\left.u\right|_{z=L}=\frac{[\cosh (a L)-1] M_{1} / T+[\sinh (a L) / a-L \cosh (a L)] G / T}{\cosh (a L)},
$$

where $a=\sqrt{T / E I}$ and $G=M_{1} a \sinh (a L) /(\cosh (a L)-1)$. The asymptotic expression for small $\alpha=a L$ (or $T<E I / L^{2}$ ) is

$$
d_{\mathrm{oc}}=\left.u\right|_{z=L}=-\frac{M_{1} L^{2}}{6 E I}\left(1+\mathcal{O}\left(\alpha^{2}\right)\right),
$$

and is valid with sufficient accuracy for a typical operational range.

Therefore, the steady state solution for strip off-centre under the asymmetric rolling conditions is obtained by simultaneous solution of equations (11) and (15), where

$$
\left.\kappa\right|_{x=L}=\left.\frac{\partial^{2} u / \partial z^{2}}{\left[1+(\partial u / \partial z)^{2}\right]^{3 / 2}}\right|_{x=L}=\frac{M_{1}}{E I}
$$

and $M_{1}=M_{1}\left(d_{\mathrm{oc}}, \omega_{1}\right.$, asymmetry) is described by the rolling model (Sections 2.1-2.3). The steady-state solution described above is obtained under the assumption that no buckling of the strip occurs. This assumption is usually reasonable if the tension applied to the strip upstream is sufficiently high. Insufficient tension results in compressive stresses acting on a part of the strip, which could lead to strip buckling. The analysis of strip buckling is beyond the scope of this paper, but it is useful to consider a simple model that supposedly captures the main features of the effect of buckling on strip tracking. Let us conjecture that the buckling makes the strip more susceptible to bending by decreasing the effective width of the strip that enters the model through the moment of inertia of the cross-section of the strip (14-15). 
By assuming, for the sake of simplicity, that buckling occurs near the concave outwards part of a bent strip where the entry stress is negative, the effective width can be calculated from the rolling model (Sections 2.1-2.3) as

$$
w_{\text {eff }}=\left\{\begin{array}{lll}
\left|\left(b_{1}, L_{s}\right)\right|, & \left\{\left(b_{1}, L_{s}\right) \subseteq\left(-L_{s}, L_{s}\right),\left.\quad \sigma_{1}(x)\right|_{x \in\left(-L_{s}, b_{1}\right)} \leq 0\right. \\
& \text { for } \left.\left.\kappa\right|_{z=L}>0\right\}, & \\
\left|\left(-L_{s}, b_{2}\right)\right|, & \left\{\left(-L_{s}, b_{2}\right) \subseteq\left(-L_{s}, L_{s}\right),\right. & \left.\sigma_{1}(x)\right|_{x \in\left(b_{2}, L_{s}\right)} \leq 0 \\
& \text { for } \left.\left.\kappa\right|_{z=L}<0\right\},
\end{array}\right.
$$

and the moment of the inertia of the cross section is

$$
I=I_{\mathrm{eff}}\left(d_{\mathrm{oc}}, \bar{\sigma}_{1}, \omega_{1}, \text { asymmetry }\right)=\frac{1}{12} \bar{h}_{1} w_{\mathrm{eff}}^{3}\left(d_{\mathrm{oc}}, \bar{\sigma}_{1}, \omega_{1}, \text { asymmetry }\right) .
$$

\section{Numerical examples and discussion}

In this section, we study two scenarios:

1. the dynamics of the strip unconstrained upstream; and

2. the steady-state tracking of the strip geometrically constrained upstream.

The data used for the calculations are as in [4].

\subsection{Dynamics of an unconstrained strip}

The dynamics of the track-off for a strip unconstrained upstream (tail out situation) is calculated using the model described in Section 2.4. It is assumed that no tension is applied to the strip upstream. The off-centre of the strip as function of time is shown in Figure 3 for two cases: a) with small 

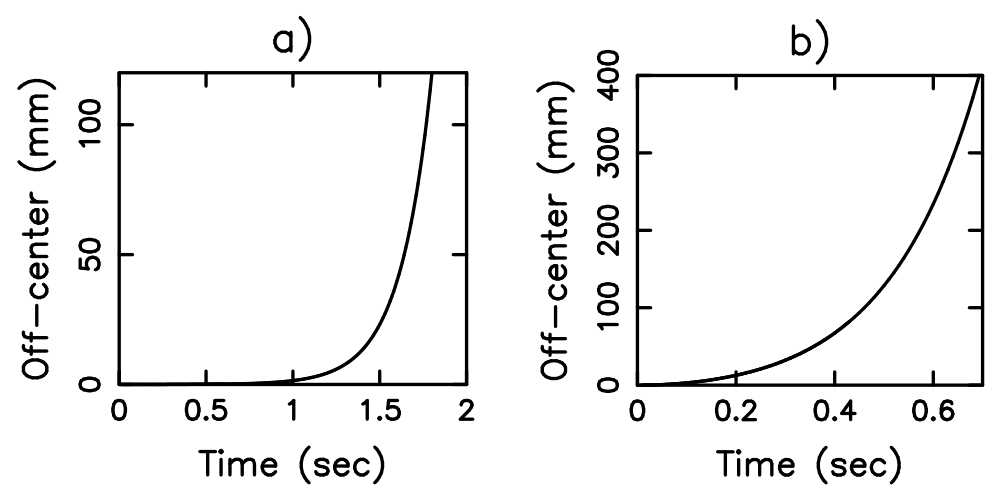

FiguRE 3: Dynamics of strip track-off for a strip with free end (a) with initial off-centre $0.01 \mathrm{~mm}$ and otherwise symmetric rolling conditions and (b) under the unequal roll forces with $\tau=0.97$.

initial off-centre but otherwise symmetric rolling conditions, and b) under the asymmetric rolling conditions, but with zero initial off-centre and entry angle. The asymmetry in case (b) is represented by the differential roll force acting on the roll stack, with the ratio of the forces on the right and left sides given by $\tau=0.97$.

See that even a small initial off-centre under symmetric rolling conditions could lead to dramatic track-off if the strip is unconstrained upstream (Figure 3a). The asymmetry in the rolling conditions significantly amplifies the rate of the track-off (Figure $3 b$ ).

\subsection{Steady state solution for a constrained strip}

The steady-state solution for a strip constrained upstream (Section 2.5) is illustrated graphically in Figure 4, where the steady-state off-centre is the horizontal coordinate of the point of intersection of the solid and dashed lines representing the solution of the rolling model and equation (11) and the 

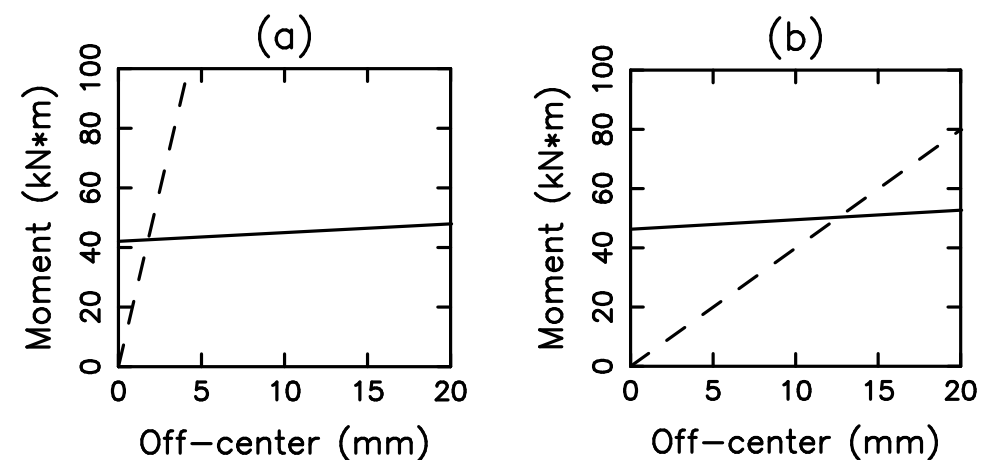

Figure 4: Graphical solution for the steady-state off-centre of the strip constrained upstream at the distance $4 \mathrm{~m}$ from the reduction roll and rolled under the unequal roll forces with $\tau=0.8$ : (a) without buckling and (b) under simplified buckling assumption.

solution of equations (14) or (15) respectively.

See in Figure 4a that the steady-state lateral deviation of the strip at the entry of the roll bite under the asymmetric rolling conditions is small if the tension applied to the strip is sufficient to prevent strip buckling. However, the buckling of the strip, according to the simplified model in Section 2.5, leads to a significant increase in the steady-state lateral deviation (Figure 4b). In principle, this could lead to the situation when the steady-state solution does not exist within the safe operational range. Thus, the importance of the applied tension and the strip buckling for strip tracking is revealed. This issue requires more rigorous study.

Acknowledgements: The authors thank the management of BHP Steel Research Laboratories for permission to publish the information contained in the paper. 


\section{References}

[1] H. Matsumoto and A. Ishii, Three-dimensional simulation of snake motion in a tandem cold mill, Iron 85 Steel Maker, volume 30(12), pages 31-39, 2003. C174, C178, C179

[2] T. Ishikawa, Y. Tozava and J. Nishizawa, Fundamental study on snaking in strip rolling, Transactions ISIJ, volume 28, pages 485-500, 1988. C174

[3] A. Nilson, FE simulations of camber in hot strip rolling, Journal of material processing technology, volumes 80-81, pages 325-329, 1998. C174

[4] T. Tarnopolskaya, F. R. de Hoog, D. J. Gates, A. Dixon and W. Y. D. Yuen, Analysis of strip track-off during flat rolling, Proceedings of 8th International Rolling Conference, volume XL, Florida, pages 237-246, 2002. C174, C175, C177, C182

[5] B. Sabatini and K. Yeomans, An algebra of strip shape and its application to mill scheduling, Journal of Iron and Steel Institute, pages 1207-1213, 1968. C176

[6] H. Matsumoto and T. Kawanami, Mechanism of material deformation related to shape and crown phenomena, Proceedings 4 th International Steel Rolling Conference, E6.1-E6.11, 1987. C176

[7] H. Matsumoto and K. Yamada, Generalised 2-dimensional theory of flat rolling and comparison with 3-dimensional FEM, Advanced Technology of Plasticity, Proc. of 5th ICTP, volume I, pages 3-6, 1996. C176

[8] H. Ford and D. R. Bland, Cold rolling with strip tension, Journal Iron and Steel Institute, pages 57-71, 1951. C176 
[9] J. H. Hitchcock, Roll-neck bearings, ASME research publications, 1935. C176

[10] W. J. Edwards and P. D. Spooner, Analysis of strip shape. In: Automation of tandem mills, G. F.Bryant (Ed.), pages 176-212, 1973. $\mathrm{C} 177$

[11] T. Tarnopolskaya and F. de Hoog, An efficient method for strip flatness analysis in cold rolling, Mathematical Engineering in Industry, 7(1), pages 71-95, 1998. C177, C178

[12] W. Y. D. Yuen, A. Duwal and B. Wechner, A new formulation for the analysis of strip flatness in strip rolling, Proceedings 7th International Conference On Steel Rolling, pages 96-301, 1998. C178 